\title{
Accuracy, conditionalization, and probabilism
}

\author{
Peter J. Lewis, University of Miami \\ Don Fallis, University of Arizona
}

March 3, 2016

\begin{abstract}
Accuracy-based arguments for conditionalization and probabilism appear to have a significant advantage over their Dutch Book rivals. They rely only on the plausible epistemic norm that one should try to decrease the inaccuracy of one's beliefs. Furthermore, it seems that conditionalization and probabilism follow from a wide range of measures of inaccuracy. However, we argue that among the measures in the literature, there are some from which one can prove conditionalization, others from which one can prove probabilism, and none from which one can prove both. Hence at present, the accuracy-based approach cannot underwrite both conditionalization and probabilism.
\end{abstract}

A central concern of epistemology is uncovering the rational constraints on an agent's credences, both at a time and over time. At a time, it is typically maintained that an agent's credences should conform to the probability axioms, and over time, it is often maintained that an agent's credences should conform to conditionalization. How could such norms be justified? The traditional approach is to show that if your credences violate these norms, then there is a set of bets, each of which you consider fair, but which collectively are such that if you accept them all you will lose money whatever happens. Since you do not want to be a "money pump", you should adopt coherent credences. However, this Dutch book strategy rests on controversial assumptions concerning prudential rationality and its connection to epistemic rationality.

The prudential elements may not be essential to the Dutch book approach (Vineberg 2012). But even so, it would be better to be able to derive probabilism and conditionalization from a clearly epistemic basic norm. A more 
recent approach seeks to do precisely that: to derive probabilism and conditionalization from the intuitive epistemic norm that you should endeavor to make your credences as accurate - as close to the truth - as possible. Drawing on the work of Joyce (1998; 2009), Greaves and Wallace (2006) and Predd et al. (2009), Pettigrew (2013) argues that the accuracy-based approach vindicates both probabilism and conditionalization. We argue that this conclusion is too strong: at present, the accuracy-based approach can vindicate either conditionalization or probabilism, but not both.

Our argument turns on the features of various proposed measures of accuracy. The accuracy-based approach is predicated on the assumption that the accuracy of your credences can be measured. Pettigrew $(2013,905)$ argues that it is a strength of the accuracy-based approach that conditionalization and probabilism follow from a wide range of measures, so that it doesn't matter which measure is used to assess the accuracy of an agent's credences. Our counter-argument is that it does matter: of the known measures, some vindicate conditionalization, and some vindicate probabilism, but there is no known measure of inaccuracy from which both conditionalization and probabilism can be derived.

\section{Accuracy and conditionalization}

First, let us briefly run through the argument via which conditionalization and probabilism are claimed to follow from considerations of accuracy, starting with conditionalization. Suppose you have credences $\mathbf{b}=\left(b_{1}, b_{2}, \ldots, b_{n}\right)$ in propositions $\mathbf{X}=\left(X_{1}, X_{2}, \ldots, X_{n}\right)$, where the propositions form a partition, i.e. they are exhaustive and mutually exclusive, so that exactly one of them is true. The accuracy approach takes it that your primary epistemic goal is having credences that are as accurate as possible, where complete accuracy is a credence of 1 in the true proposition and a credence of 0 in each of the false propositions. The closer your credences are to complete accuracy, the better.

For this epistemic goal to make sense, we need a measure of closeness. In what follows we will discuss several such measures, expressed as measures of inaccuracy: the larger the measure, the further your credences are from the truth. Hence your goal is to minimize the value of this inaccuracy measure. By far the dominant measure in the literature is the quadratic rule or Brier rule, which takes the square of the difference between your credence in each 
proposition and its truth value, and sums the results. So for a partition, if $I_{i}(\mathbf{b})$ is the inaccuracy of credences $\mathbf{b}$ when proposition $X_{i}$ is true, then the Brier rule can be expressed as follows: ${ }^{1}$

Simple Brier rule: $I_{i}(\mathbf{b})=\left(1-b_{i}\right)^{2}+\sum_{j \neq i} b_{j}^{2}$.

The Brier rule has been defended by epistemologists (Joyce 2009, 290; Leitgeb and Pettigrew 2010,219), and is frequently cited as the prime example of an inaccuracy measure (Greaves and Wallace 2006, 627; Pettigrew 2013, 899).

Suppose you obtain evidence $E$ that is consistent with some but not all of the propositions $\mathbf{X}$. How should you distribute your credence over the remaining propositions? If your goal is to minimize your inaccuracy, presumably the best you can do is to minimize your expected inaccuracy given your prior credences $\mathbf{b}$. So suppose that after you learn $E$, you shift your credence in proposition $X_{i}$ from $b_{i}$ to $x$. If $X_{i}$ is true, the contribution of this new credence to your overall inaccuracy is $(1-x)^{2}$, and if $X_{i}$ is false, the contribution is $x^{2}$. Given your prior credences $\mathbf{b}$, you judge that the chance that $X_{i}$ is true is $b_{i}$, and the chance that $X_{i}$ is false is $\sum_{E-i} b_{j}$, where the notation $E-i$ indicates that the sum is over all propositions consistent with $E$ except $X_{i}$. That is, the total contribution $C$ of this new credence to your expected inaccuracy is given by:

$$
C=(1-x)^{2} b_{i}+x^{2} \sum_{E-i} b_{j} .
$$

Your goal is to minimize $C$. So consider where $d C / d x=0$ :

$$
\begin{aligned}
\frac{d C}{d x} & =-2(1-x) b_{i}+2 x \sum_{E-i} b_{j} \\
& =-2 b_{i}+2 x \sum_{E} b_{j}
\end{aligned}
$$

where the sum in the last line is now over all propositions consistent with $E$. This expression is zero when

$$
x=\frac{b_{i}}{\sum_{E} b_{j}} .
$$

\footnotetext{
${ }^{1}$ We call the version of the Brier rule applicable to a partition the simple Brier rule only for ease of reference (and similarly for the simple log rule and simple spherical rule to be introduced later).
} 
But note that this value for $x$ is just your prior credence in $X_{i}$ conditional on $E$ :

$$
c\left(X_{i} \mid E\right)=\frac{c\left(X_{i} \wedge E\right)}{c(E)}=\frac{b_{i}}{\sum_{E} b_{j}} .
$$

That is, conditionalizing on $E$ minimizes your expected inaccuracy. ${ }^{2}$ So if your epistemic goal is to minimize inaccuracy, you should conditionalize on new evidence.

Greaves and Wallace (2006) generalize this proof to cover measures of inaccuracy other than the Brier rule. In particular, they show that conditionalization minimizes expected inaccuracy for any measure of inaccuracy $I_{i}(\mathbf{b})$ satisfying strict propriety:

Strict propriety: For any distinct probabilistic credences $\mathbf{b}$ and $\mathbf{b}^{\prime}, \sum_{i} b_{i} I_{i}(\mathbf{b})<$ $\sum_{i} b_{i} I_{i}\left(\mathbf{b}^{\prime}\right)$.

Strict propriety says that the expected inaccuracy of your current credences $\mathbf{b}$ is lower than the expected inaccuracy of any alternative credences $\mathbf{b}^{\prime}$ you might adopt, where the expectation is calculated according to your current credences. If it fails, then the injunction to minimize inaccuracy makes your beliefs pathologically unstable: you can lower your expected inaccuracy by shifting your credences, even in the absence of new evidence. Hence strict propriety serves as a reasonable constraint on measures of inaccuracy. The Brier rule is strictly proper, as are several other proposed inaccuracy measures to be discussed below.

Greaves and Wallace begin by introducing some terminology. They say that a set of credences $\mathbf{b}$ recommends a set of credences $\mathbf{b}^{\prime}$ iff the expected inaccuracy of $\mathbf{b}^{\prime}$ is at least as low as the expected inaccuracy of $\mathbf{b}$, where the expectation is calculated using credences $\mathbf{b}$ :

Recommendation: $\mathbf{b}$ recommends $\mathbf{b}^{\prime}$ iff $\sum_{i} b_{i} I_{i}(\mathbf{b}) \geq \sum_{i} b_{i} I_{i}\left(\mathbf{b}^{\prime}\right)$

Note that if the inaccuracy measure $I_{i}(\mathbf{b})$ satisfies strict propriety, then $\mathbf{b}$ only recommends itself.

They further define quasi-conditionalization as a belief updating rule that stipulates that your credences on learning $E$ should be some set recommended by your prior credences conditional on $E$. They then prove

\footnotetext{
${ }^{2}$ This proof is a simplified version of the one in Leitgeb and Pettigrew (2010).
} 
that quasi-conditionalization is always optimal: whatever measure of inaccuracy you choose, strictly proper or not, the expected inaccuracy of quasiconditionalizing is at least as low as the expected inaccuracy of any other updating rule. Then if your measure of inaccuracy is strictly proper, conditionalization itself is optimal, since for strictly proper measures, credences only recommend themselves. In fact, since the inequality in strict propriety

is strict, conditionalization is strictly better than any other updating rule: it uniquely minimizes expected inaccuracy. As Pettigrew (2013, 905) notes, this is a strong result: any inaccuracy measure satisfying strict propriety can be used to vindicate conditionalization, and strict propriety is a constraint we would expect any reasonable inaccuracy measure to obey anyway.

\section{Accuracy and probabilism}

Now let us turn to the arguments that your credences at a time should obey the probability axioms. So far, we have been assuming that the propositions we are interested in form a partition. But the probability axioms include constraints on your credences in disjunctions, and to model such constraint we need to allow that more than one of the propositions you are considering can be true. To that end, suppose that you have credences $\mathbf{b}=\left(b_{1}, b_{2}, \ldots, b_{n}\right)$ in propositions $\mathbf{X}=\left(X_{1}, X_{2}, \ldots, X_{n}\right)$, where now the set of propositions forms a Boolean algebra, i.e. it is closed under negation and disjunction. So now we can no longer model a possible world simply as an index (picking out the unique true proposition); instead, we need to label each proposition separately as either true or false. That is, a possible world is specified by $\boldsymbol{\omega}=\left(\omega_{1}, \omega_{2}, \ldots \omega_{n}\right)$, where $\omega_{i}=1$ when $X_{i}$ is true and $\omega_{i}=0$ when $X_{i}$ is false. In this context, the Brier rule can be rewritten as follows:

Symmetric Brier rule: $I(\boldsymbol{\omega}, \mathbf{b})=\sum_{i}\left(b_{i}-\omega_{i}\right)^{2}$.

As before, the inaccuracy of your beliefs according to the Brier rule is given by the sum of the squares of the distance of each belief from the relevant truth value. That is, the Brier rule is symmetric, in the sense that distance from the truth for a true proposition plays the same role as distance from falsity plays for a false proposition. This property will be important later.

The general strategy for defending probabilism based on accuracy goes as follows. Suppose that your current credences are incoherent - that is, they 


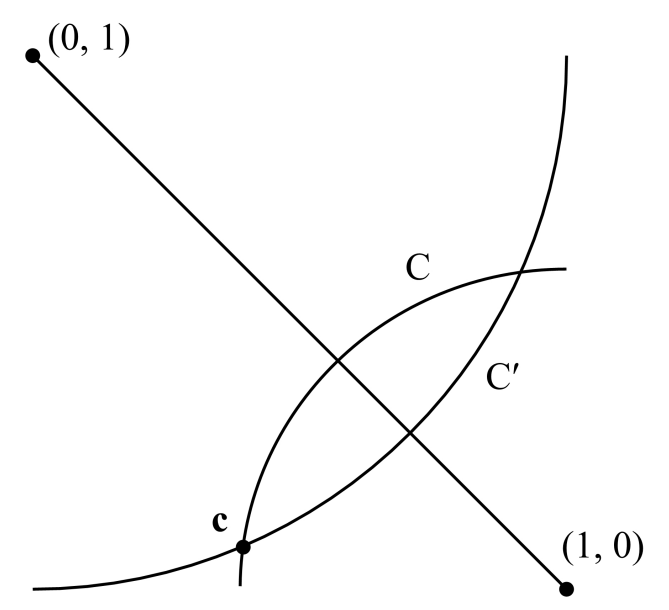

Figure 1: De Finetti's construction for a two-element partition (Joyce 1998, $582)$.

violate the probability axioms. Then one can appeal to a measure of inaccuracy to show that there are coherent credences that dominate your current credences - that are more accurate than your current credences whatever the truth values of the propositions concerned. If your goal is to minimize inaccuracy, this gives you a clear reason to avoid incoherent credences: there are always coherent credences that are more accurate, whatever the world is like.

De Finetti $(1974,87)$ constructs a dominance argument of this kind based on the Brier rule. ${ }^{3}$ For illustration, consider the simple case of a proposition and its negation: that is, the propositions under consideration are just $(X, \neg X)$. In this case the space of possible credences forms a plane, as shown in figure 1: your credence in $X$ is the horizontal coordinate, and your credence in $\neg X$ is the vertical coordinate. The two possible worlds are represented by the points $(1,0)$ and $(0,1)$, and your credences obey the probability axioms if and only if they lie on the straight line that connects these two points, since along this line your credences in $X$ and $\neg X$ sum to 1 .

Suppose that your credences are incoherent: they are represented by a point $\mathbf{c}=\left(c_{1}, c_{2}\right)$ that lies off this diagonal. And suppose first that the

\footnotetext{
${ }^{3}$ As Joyce $(1998,580)$ notes, de Finetti sets up this argument in terms of bets. However, as Pettigrew $(2013,901)$ points out, it can be redescribed as an accuracy-based argument.
} 
actual world is represented by the bottom-right corner $(1,0)$-i.e. $X$ is true and $\neg X$ is false. Then the inaccuracy of your credences according to the Brier rule is $I(\boldsymbol{\omega}, \mathbf{c})=\left(1-c_{1}\right)^{2}+\left(c_{2}\right)^{2}$. Note that this is just the square of the Euclidean distance between $\left(c_{1}, c_{2}\right)$ and $(1,0)$. That is, every point on the circle segment $C$ has the same inaccuracy as $\mathbf{c}$, and every point between $C$ and $(1,0)$ has a lower inaccuracy. Now suppose instead that the actual world is represented by the top-left corner $(0,1)$-i.e. $X$ is false and $\neg X$ is true. Then the inaccuracy of your credences is $I(\boldsymbol{\omega}, \mathbf{c})=\left(c_{1}\right)^{2}+\left(1-c_{2}\right)^{2}$ - the square of the Euclidean distance between $\left(c_{1}, c_{2}\right)$ and $(0,1)$. That is, every point on the circle segment $C^{\prime}$ has the same inaccuracy as $\mathbf{c}$, and every point between $C$ and $(0,1)$ has a lower inaccuracy.

Consider the area enclosed by the circle segments $C$ and $C^{\prime}$. The credences represented by the points in this area have a lower inaccuracy than $\mathbf{c}$ if $X$ is true and $\neg X$ false, and a lower inaccuracy than $\mathbf{c}$ if $X$ is false and $\neg X$ true. That is, they have a lower inaccuracy whatever the world is like. And this area includes part of the diagonal that represents coherent credences. So for any incoherent set of credences, there is a coherent set that is less inaccurate whatever the world is like. In this simple case, accuracy gives you a motive to adopt coherent credences.

In the general case, the space of possible credences is $n$-dimensional, where there are $n$ propositions in the Boolean algebra. Each possible assignment of truth values to the $n$ propositions is represented by a point in this space, and the set of coherent credences consists of these points plus the points on the straight lines that connect them, the points on the straight lines that connect those latter points, and so on. This set is called the convex hull $V^{+}$of the possible truth value assignments $V$. Via a generalization of the construction of figure 1, de Finetti shows that if your credences are represented by a point that lies outside $V^{+}$, then there are points in $V^{+}$ that are more accurate (according to the Brier rule) whichever point in the space represents the actual truth values of the propositions. Hence if you have incoherent credences, there are always coherent credences with a lower inaccuracy as measured by the Brier rule.

Predd et al. (2009) generalize this proof strategy to cover a wider range of inaccuracy measures. Their proof relies on two assumptions. The first is additivity:

Additivity: $I(\boldsymbol{\omega}, \mathbf{b})$ can be expressed as $\sum_{i} s\left(\omega_{i}, b_{i}\right)$, where $s$ is a continuous function of your credence in proposition $X_{i}$ and its truth value. 
Additivity states that the inaccuracy of your beliefs in a set of propositions is just the sum of your inaccuracies in the propositions taken individually - that is, $s\left(\omega_{i}, b_{i}\right)$ is the inaccuracy of your belief in proposition $X_{i}$, and $I(\boldsymbol{\omega}, \mathbf{b})$ is just the sum of these inaccuracies for all the propositions you are considering. Note that it also contains the requirement that the inaccuracy measure should be continuous. The Brier rule is obviously additive, since it is expressed as a sum over propositions.

The second assumption is a version of strict propriety. For an additive inaccuracy measure, strict propriety can be expressed in terms of your inaccuracy function for a single proposition $s\left(b_{i}, \omega_{i}\right)$ as follows:

Strict propriety (for an additive measure): $b_{i} s(x, 1)+\left(1-b_{i}\right) s(x, 0)$ is uniquely minimized at $x=b_{i}$.

Predd et al. (2009) prove that any additive, strictly proper inaccuracy measure entails probabilism. De Finetti's construction appeals to the natural distance measure implicit in the Brier rule - the Euclidean distance between two points in the space of your possible credences. But in the current case we have no explicit measure of inaccuracy, so Predd et al. appeal to a generalized "distance" measure ${ }^{4}$ called the Bregman divergence, defined for a strictly convex function $\Phi(\mathbf{x})$ as $d_{\Phi}(\mathbf{y}, \mathbf{x})=\Phi(\mathbf{y})-\Phi(\mathbf{x})-\nabla \Phi(\mathbf{x}) \cdot(\mathbf{y}-\mathbf{x})$. They show that if the inaccuracy measure $s\left(b_{i}, \omega_{i}\right)$ for a single proposition $X_{i}$ is strictly proper, then the function $\varphi\left(b_{i}\right)=-b_{i} s\left(b_{i}, 1\right)-\left(1-b_{i}\right) s\left(b_{i}, 0\right)$ is strictly convex. In terms of this function, Predd et al. show that for any additive, strictly proper inaccuracy measure, $I(\boldsymbol{\omega}, \mathbf{b})=d_{\Phi}(\boldsymbol{\omega}, \mathbf{b})$, where $\Phi(\boldsymbol{\omega})=\sum_{i} \varphi\left(\omega_{i}\right)$ and $\Phi(\mathbf{b})=\sum_{i} \varphi\left(b_{i}\right)$.

The set of coherent credences forms a closed, convex subspace $V^{+}$of the space of all possible credences. It is a fact from the theory of Bregman divergences that for any point $\mathbf{c}$ outside $V^{+}$, there is a unique point $\mathbf{c}^{*}$ in $V^{+}$such that $d_{\Phi}\left(\mathbf{c}^{*}, \mathbf{c}\right) \leq d_{\Phi}(\mathbf{y}, \mathbf{c})$ for all $\mathbf{y}$ in $V^{+}$. That is, $\mathbf{c}^{*}$ is the unique closest point in $V^{+}$to $\mathbf{c}$, using the Bregman divergence as a distance measure. It is a further fact that $d_{\Phi}\left(\mathbf{y}, \mathbf{c}^{*}\right) \leq d_{\Phi}(\mathbf{y}, \mathbf{c})-d_{\Phi}\left(\mathbf{c}^{*}, \mathbf{c}\right)$ for all $\mathbf{y}$ in $V^{+}$and c outside $V^{+}$. Note in particular that $V^{+}$contains every possible world $\boldsymbol{\omega}$, since a consistent truth value assignment is also a coherent set of credences. So setting $\mathbf{y}=\boldsymbol{\omega}$, we have $d_{\Phi}\left(\boldsymbol{\omega}, \mathbf{c}^{*}\right) \leq d_{\Phi}(\boldsymbol{\omega}, \mathbf{c})-d_{\Phi}\left(\mathbf{c}^{*}, \mathbf{c}\right)$. Since $d_{\Phi}$ is a positive-valued function, $d_{\Phi}\left(\mathbf{c}^{*}, \mathbf{c}\right)>0$, so $d_{\Phi}\left(\boldsymbol{\omega}, \mathbf{c}^{*}\right)<d_{\Phi}(\boldsymbol{\omega}, \mathbf{c})$, and hence

\footnotetext{
${ }^{4}$ The reason for the scare quotes is that the Bregman divergence is not symmetric, and distance measures are typically symmetric.
} 
$I\left(\boldsymbol{\omega}, \mathbf{c}^{*}\right)<I(\boldsymbol{\omega}, \mathbf{c})$. That is, for any incoherent set of credences $\mathbf{c}$, there is a coherent set $\mathbf{c}^{*}$ that is less inaccurate than $\mathbf{c}$ in every possible world.

As Pettigrew $(2013,905)$ notes, this is a strong result: any inaccuracy measure satisfying strict propriety and additivity can be used to vindicate probabilism, and while additivity is perhaps not forced on us in the way that strict propriety is, it is certainly intuitive. As we shall see, there are several available measures satisfying additivity and strict propriety, so it initially looks like the accuracy-based program can justify both probabilism and conditionalization based on minimal premises. Our purpose in this paper is to argue that matters are not so straightforward.

\section{$3 \quad$ Measures of inaccuracy}

Let us return to the argument for conditionalization. This argument restricts inaccuracy measures to those that are strictly proper. Note that strict propriety is only a condition on expected inaccuracy. But expected inaccuracy is calculated on the basis of the actual inaccuracy that the measure in question ascribes to credences, and presumably there are a number of constraints any such measure must obey if it is to genuinely measure epistemic inaccuracy rather than something else. For example, if one of your credences shifts towards the truth, while your other credences stay the same, then clearly your actual inaccuracy should decrease. We wish to focus on one such constraint.

The constraint can be motivated by thinking about elimination cases. Suppose you are considering a set of mutually exclusive and exhaustive propositions, and suppose that your credences are coherent and that you conditionalize on evidence. You acquire some evidence that eliminates one false proposition - your credence in it becomes zero - but is uninformative regarding the other hypotheses - your credences in them remain in the same proportions. How does this affect the accuracy of your credences?

It seems obvious that your beliefs have become more accurate. If you believe that Tom, Dick or Harry might be the murderer (when in fact Tom did it), and you eliminate Harry while learning nothing about Tom or Dick, then you have made epistemic progress towards the truth, or at least away from falsity. It is true that your credence in the false proposition "Dick did it" goes up, but only by the same proportion that your credence in the true proposition "Tom did it" goes up.

Unfortunately, the simple Brier rule does not always concur. Let $X_{1}$ be 
"Tom did it", $X_{2}$ be "Dick did it", and $X_{3}$ be "Harry did it", where unknown to you $X_{1}$ is true. Suppose that your initial credences in $\left(X_{1}, X_{2}, X_{3}\right)$ are $\mathbf{b}=(1 / 7,3 / 7,3 / 7)$. Then according to the simple Brier rule, your initial inaccuracy is $54 / 49=1.10$. Now suppose you acquire some evidence that eliminates $X_{3}$, but is uninformative regarding $X_{1}$ and $X_{2}$. That is, your credence in $X_{3}$ becomes 0 and your credences in $X_{1}$ and $X_{2}$ stay in the same proportions, so that your final credences are $\mathbf{b}^{*}=(1 / 4,3 / 4,0)$. Then according to the simple Brier rule, your final inaccuracy is $18 / 16=1.13$. That is, the Brier rule erroneously says that the inaccuracy of your beliefs has gone up.

For a measure to genuinely measure the actual inaccuracy of your beliefs, it should not be susceptible to counterexamples of this kind; it should count elimination cases as epistemically positive. That is, measures of inaccuracy should obey the following principle:

M: For coherent credences over a partition, if $\mathbf{b}$ assigns a zero credence to some false proposition to which $\mathbf{b}^{\prime}$ assigns a non-zero credence, and credences in the remaining propositions stay in in the same ratios, then $\mathbf{b}$ is epistemically better than $\mathbf{b}^{\prime}$.

The simple Brier rule, as the example shows, violates $\mathrm{M}$, and hence does not plausibly measure the actual inaccuracy of your beliefs. ${ }^{5}$

Fortunately, though, there are alternative inaccuracy measures for partitions we can appeal to. The two most frequently mentioned are the simple log rule and the simple spherical rule:

Simple log rule: $I_{i}(\mathbf{b})=-\ln b_{i}$

Simple spherical rule: $I_{i}(\mathbf{b})=1-b_{i} / \sqrt{\sum_{j} b_{j}^{2}}$.

As before, $I_{i}(\mathbf{b})$ is the inaccuracy of credences $\mathbf{b}$ when proposition $X_{i}$ is true. Both of these measures satisfy $\mathrm{M}$, and hence are not susceptible to elimination counterexamples. ${ }^{6}$ Hence each can plausibly be claimed to measure epistemic inaccuracy. Furthermore, each is strictly proper, and so each can be used to

\footnotetext{
${ }^{5}$ One might reasonably think that acceptable measures of accuracy should obey a stronger principle than $\mathrm{M}$; see (reference removed).

${ }^{6}$ This is trivial for the log rule, and easily proven for the spherical rule. See (reference removed).
} 
underwrite conditionalization via the above argument strategy. So there are some inaccuracy measures that vindicate conditionalization, but not all strictly proper measures do so. In particular, the simple Brier rule cannot be used to vindicate conditionalization.

But what about probabilism? The simple log rule and simple spherical rule are not applicable to a Boolean algebra, and so cannot be used to prove probabilism as they stand. Perhaps the most straightforward way to generalize them is simply to sum the contribution given by the simple rule for each true proposition in the Boolean algebra, while ignoring the false propositions in the algebra:

Asymmetric log rule: $I(\mathbf{b}, \boldsymbol{\omega})=\sum_{i} F\left(\omega_{i}, b_{i}\right)$, where $F\left(0, b_{i}\right)=0$ and $F\left(1, b_{i}\right)=-\ln b_{i}$.

Asymmetric spherical rule: $I(\mathbf{b}, \boldsymbol{\omega})=\sum_{i} F\left(\omega_{i}, b_{i}\right)$, where $F\left(0, b_{i}\right)=0$ and $F\left(1, b_{i}\right)=1-b_{i} / \sqrt{\sum_{j} b_{j}^{2}}$.

Both these rules are asymmetric, in the sense that inaccuracy is calculated differently for true and false propositions. These rules satisfy principle M: for coherent credences, if your credence in a false proposition goes down and your remaining credences stay in the same ratios, then your credence in each true proposition goes up, and so your inaccuracy according to the relevant asymmetric rules goes down. Hence the asymmetric log and spherical rules are immune from elimination counterexamples.

But these rules do not satisfy the combination of additivity and strict propriety required for the proof of probabilism. The asymmetric spherical rule is not additive: $F\left(1, b_{i}\right)$ is not a function of $b_{i}$ alone. The asymmetric log rule is additive, but it is not strictly proper in the required sense: $F\left(1, b_{i}\right)$ is strictly proper, but $F\left(0, b_{i}\right)$ is not. Indeed, it is straightforward to show directly that these rules cannot be used as the basis of a dominance argument for probabilism. Consider, for example, a two element partition, and the incoherent credence assignment $(1,1)$. The asymmetric log rule counts these incoherent credences as perfectly accurate (since the credence in the false proposition is ignored), so no coherent credences can dominate them. According to the asymmetric spherical rule, multiplying all credences by a constant has no effect on inaccuracy, so this assignment has the same inaccuracy as the coherent credence assignment $(1 / 2,1 / 2)$. If coherent assignments cannot be dominated, then neither can the initial incoherent assignment. 
But if coherent assignments can be dominated then the dominance proof of probabilism fails anyway.

So the asymmetric versions of the log rule and the spherical rule cannot be used to prove probabilism. But for a Boolean algebra, the log rule and the spherical rule are usually given a formulation that is symmetric between truth and falsity:

Symmetric log rule: $I(\boldsymbol{\omega}, \mathbf{b})=\sum_{i}-\ln \left|\left(1-\omega_{i}\right)-b_{i}\right|$

Symmetric spherical rule: $I(\boldsymbol{\omega}, \mathbf{b})=\sum_{i} 1-\frac{\left|\left(1-\omega_{i}\right)-b_{i}\right|}{\sqrt{b_{i}^{2}+\left(1-b_{i}\right)^{2}}}$

(see e.g. Joyce 2009, 275). These measures are additive, and each term in the sum is individually strictly proper, so they can each be used to prove probabilism via the proof of Predd et al.

But unfortunately, in their symmetric forms all three rules-Brier, log and spherical - are subject to elimination counterexamples. For the Brier rule, the counterexample is the same as before, since the symmetric Brier rule reduces to the simple Brier rule when applied to a partition. ${ }^{7}$ That is, consider a credence shift from $\mathbf{b}=(1 / 7,3 / 7,3 / 7)$ to $\mathbf{b}^{*}=(1 / 4,3 / 4,0)$ when $X_{1}$ is true. According to the symmetric Brier rule, your initial inaccuracy is 1.10 , and your final inaccuracy is 1.13, so your inaccuracy goes up. And this example works equally well against the symmetric spherical rule: according to this rule, your initial inaccuracy is 1.24 and your final inaccuracy is 1.37 , so your inaccuracy goes up. This particular counterexample does not work against the symmetric log rule, but a similar one does. Suppose your initial credences are $\mathbf{b}=(1 / 13,6 / 13,6 / 13)$, and your final credences are $\mathbf{b}^{*}=(1 / 7,6 / 7,0)$. Then according to the symmetric log rule your initial inaccuracy is 3.80 , and your final inaccuracy is 3.89: your inaccuracy goes up. Hence the symmetric measures all violate principle $\mathrm{M}$, and so none of them can be used to prove conditionalization.

\footnotetext{
${ }^{7}$ Strictly, applying these rules to a Boolean algebra requires including credences in the negations $\neg X_{1}, \neg X_{2}$ and $\neg X_{3}$, plus the tautology $X_{1} \vee X_{2} \vee X_{3}$ and the contradiction $\neg\left(X_{1} \vee X_{2} \vee X_{3}\right)$. But for coherent credences the inaccuracies of the tautology and the contradiction are zero, and for symmetric rules the inaccuracy of $\neg X_{i}$ is the same as that of $X_{i}$, so the inaccuracy calculated over the entire Boolean algebra is simply twice the inaccuracy over the partition $\left(X_{1}, X_{2}, X_{3}\right)$.
} 


\section{The extent of the problem}

Let us sum up. The simple Brier rule cannot be used to prove conditionalization, but the simple log and spherical rules can. The obvious generalizations of the simple log and spherical rules to a Boolean algebra - the asymmetric log and spherical rules - cannot be used to prove probabilism. The symmetric Brier, log and spherical rules can be used to prove probabilism, but none of them underwrites conditionalization. So we have found no measure that can be used to prove both conditionalization and probabilism.

Could there be such a measure? Perhaps, although it is worth noting that one can prove that any inaccuracy measure that satisfies additivity, strict propriety and a plausible symmetry principle is subject to elimination counterexamples. The symmetry principle is precisely the one discussed above - that the inaccuracy measure treats truth the same as falsity, in the sense that it is a function of the distance between each credence and its respective truth value. For an additive inaccuracy measure, the symmetry principle can be expressed in terms of the inaccuracy function for a single proposition $s\left(\omega_{i}, b_{i}\right)$ as follows:

Symmetry: $s\left(\omega_{i}, b_{i}\right)=s\left(\left|1-\omega_{i}\right|,\left|1-b_{i}\right|\right)$.

It is certainly highly plausible that this is part of what it means for $s$ to measure your distance from the truth, and as discussed above, the typical Boolean algebra forms of the Brier rule, log rule and spherical rule all satisfy it.

Let us see how this symmetry principle, together with additivity and strict propriety, lead to elimination counterexamples. Consider a single proposition $X_{i}$ in which your credence is $b_{i}=1 / 2$. According to strict propriety, the quantity $(1 / 2) s(1, x)+(1 / 2) s(0, x)$ must be uniquely minimized at $x=1 / 2$. In particular, the value of this expression for $x=1 / 2$ must be lower than its value for $x=1$ :

$$
(1 / 2) s(1,1 / 2)+(1 / 2) s(0,1 / 2)<(1 / 2) s(1,1)+(1 / 2) s(0,1),
$$

and for $x=0$ :

$$
(1 / 2) s(1,1 / 2)+(1 / 2) s(0,1 / 2)<(1 / 2) s(1,0)+(1 / 2) s(0,0) .
$$

Adding these:

$s(1,1 / 2)+s(0,1 / 2)<(1 / 2) s(1,1)+(1 / 2) s(0,1)+(1 / 2) s(1,0)+(1 / 2) s(0,0)$. 
But by symmetry, $s(1,1 / 2)=s(0,1 / 2), s(1,1)=s(0,0)$ and $s(0,1)=s(1,0)$. Substituting:

$$
2 s(0,1 / 2)<s(0,1)+s(0,0) .
$$

Now consider your credences in three exhaustive and mutually exclusive propositions $\mathbf{X}=\left(X_{1}, X_{2}, X_{3}\right)$. Consider in particular the credence shift from $\mathbf{m}=(0,1 / 2,1 / 2)$ to $\mathbf{b}=(0,1,0)$ for truth values $\boldsymbol{\omega}=(1,0,0)$. By separability, $I(\boldsymbol{\omega}, \mathbf{m})=s(1,0)+2 s(0,1 / 2)$, and $I(\boldsymbol{\omega}, \mathbf{b})=s(1,0)+s(0,1)+$ $s(0,0)$. So since $2 s(0,1 / 2)<s(0,1)+s(0,0)$ it follows that $I(\boldsymbol{\omega}, \mathbf{m})<$ $I(\boldsymbol{\omega}, \mathbf{b})$ : your inaccuracy goes up. But the shift from $\mathbf{m}=(0,1 / 2,1 / 2)$ to $\mathbf{b}=(0,1,0)$ is an elimination case: a false proposition is eliminated, and your credences in the remaining hypotheses stay in the same proportions. And lest one worry about the fact that your initial credence in the true proposition is zero, we can modify the example. Consider the credence assignments $\mathbf{m}^{\prime}=(\delta /(2+\delta), 1 /(2+\delta), 1 /(2+\delta))$ and $\mathbf{b}^{\prime}=(\delta /(1+\delta), 1 /(1+\delta), 0)$. For small $\delta$ these are close to $\mathbf{m}$ and $\mathbf{b}$, and hence by the continuity clause of additivity, the inaccuracy of $\mathbf{m}^{\prime}$ remains lower than that of $\mathbf{b}^{\prime}$. Again, the transition from $\mathbf{m}^{\prime}$ to $\mathbf{b}^{\prime}$ is an elimination case, and now your credence in the true proposition is non-zero.

So elimination counterexamples afflict any inaccuracy measure that satisfies additivity, strict propriety and symmetry. That is, any symmetric measure that satisfies the assumptions of Predd et al.'s proof of probabilism violates principle $\mathrm{M}$, and hence cannot be used to prove conditionalization. Symmetry is not a premise in the Predd argument, so it is possible that an asymmetric measure might allow the derivation of both probabilism and conditionalization. But the only plausible asymmetric measure in the literature is the log rule (Bernardo 1979), and we have seen that the asymmetric log rule does not vindicate probabilism.

\section{Conclusion}

Pettigrew notes that conditionalization and probabilism follow from a wide range of measures of inaccuracy, and the implication is that it doesn't much matter which measure you pick. But we think it does matter. There are measures that vindicate conditionalization, and there are measures that vindicate probabilism, but nobody has yet identified a measure that vindicates both. Hence the accuracy-based approach does not, as yet, give us the justification we might want for the constraints on our credences. 


\section{References}

Bernardo, José M. (1979), "Expected information as expected utility", Annals of Statistics 7: 686-690.

de Finetti, Bruno (1974), Theory of Probability, vol. 1. New York: John Wiley and Sons.

Greaves, Hilary and David Wallace (2006), "Justifying conditionalization: conditionalization maximizes expected epistemic utility", Mind 115: $607-32$.

Joyce, James M.. (1998), "A nonpragmatic vindication of probabilism", Philosophy of Science, 65: 575-603.

Joyce, James M. (2009), "Accuracy and coherence: prospects for an alethic epistemology of partial belief", in F. Huber and C. Schmidt-Petri (eds.), Degrees of Belief. Dordrecht: Springer: 263-97.

Leitgeb, Hannes, and Richard Pettigrew (2010), "An objective justification of Bayesianism I: measuring inaccuracy", Philosophy of Science 77: 201-35.

Pettigrew, Richard (2013), "Epistemic utility and norms for credence", Philosophy Compass 8: 897-908.

Predd, Joel B., Robert Seiringer, Elliott H. Lieb, Daniel N. Osherson, H. Vincent Poor, and Sanjeev R. Kulkarni (2009), "Probabilistic coherence and proper scoring rules", IEEE Transactions on Information Theory 55: $4786-4792$.

Vineberg, Susan (2012), "Dutch book arguments", in Edward N. Zalta (ed.), Stanford Encyclopedia of Philosophy. http://plato.stanford.edu/ archives/spr2012/entries/dutch-book/ 